\title{
LEVAMISOL NÃO PREVINE LESÕES DE ESTOMATITE AFTOSA RECORRENTE"' UM ENSAIO CLÍNICO RANDOMIZADO, DUPLO-CEGO E CONTROLADO POR PLACEBO
}

\author{
Luc Louis Maurice Weckx*1, Cleonice Hitomi Watashi Hirata², Marilda Aparecida Milanez Morgado de Abreu*3, Vilma Ciorla Fillizolla ${ }^{4}$, \\ Olga Maria Panhoca da Silva ${ }^{5}$ \\ Trabalho realizado na Universidade Federal de São Paulo - UNIFESP, S. Paulo, SP
}

\author{
*Correspondência: \\ Disciplina de \\ Otorrinolaringologia \\ Rua dos Otonis, 684 \\ V. Clementino \\ CEP 04025-001 \\ (11) $5539-7723$ \\ lucweckx@uol.com.br
}

\begin{abstract}
RESUMO
Oвjetivo. Avaliar a eficácia e a segurança do levamisol no tratamento profilático da afta recorrente, utilizando um protocolo de estudo duplo-cego.

Métodos. Quatorze pacientes receberam doses decrescentes de levamisol por via oral por seis meses (dose inicial de $150 \mathrm{mg}$ três vezes por semana). Dez pacientes receberam placebo. As avaliações foram mensais.

Resultados. Houve tendência à diminuição do número de crises nos dois grupos, mas sem diferenças entre ambos. 0 número de lesões diminuiu significantemente nos grupos levamisol e placebo, mas na comparação entre eles a diferença não foi significante. A duração das lesões diminuiu significantemente no grupo placebo, porém ao compará-lo com o grupo levamisol a diferença não foi significante durante todo o tratamento. A intensidade da dor foi significantemente menor nos dois grupos, mas ao compará-los a dor foi significantemente menor no grupo placebo. A avaliação global final mostrou melhora em $50 \%$ dos pacientes do grupo levamisol e em $70 \%$ do Placebo, sem diferença significante entre os dois tratamentos. Não foi observada diferença na frequência de efeitos colaterais entre os grupos.
\end{abstract}

Conclusão. Levamisol, como usado nesse protocolo, é uma droga segura. Comparado ao placebo, levamisol não é efetivo no tratamento profilático da afta recorrente. 0 efeito placebo é importante em desordens nas quais fatores emocionais afetam a recorrência ou a expressão de sintomas.

Uniterm os: Efeito placebo. Ensaio clínico controlado aleatório. Estomatite aftosa. Levamisol. Terapia.

\section{INTRODUÇÃo}

Afta recorrente é uma doença comum que se caracteriza por episódios recorrentes de lesões ulcerativas na mucosa da cavidade oral. Embora seu curso clínico seja benigno, causa dor e desconforto, com comprometimento da qualidade de vida. Acomete homens e mulheres de todas as idades, raças e regiões geográficas, com uma prevalência estimada em até $25 \%$ da população. Embora se desconheça a causa exata da afta recorrente, é sugerido que muitos fatores locais e sistêmicos, tais como trauma local, estresse, ingestão de certos alimentos, drogas, alterações hormonais, deficiência de vitaminas e agentes infecciosos estejam associados à doença. Há suspeitas do envolvimento de fatores genéticos e imunológicos. Não há tratamento curativo preconizado; os corticosteróides levam à regressão da crise, mas todas as modalidades terapêuticas disponíveis para a prevenção apenas reduzem a frequência ou a gravidade das lesões. Para isso, várias drogas (Colchicina, talidomida, pentoxifilina, dapsona, levamisol e outras) têm sido utilizadas, porém a maioria não demonstrou eficácia comprovada ou não foi avaliada por estudos clínicos controlados. 0 emprego da maioria dessas drogas é baseado nas suas propriedades anti-inflamatórias e imunomoduladoras, visto que a afta recorrente é uma doença inflamatória aparentemente relacionada a anormalidades do sistema imune $e^{1-3}$.

O levamisol é um anti-helmíntico que possui propriedades imunomoduladoras, o que poderia justificar sua efetividade no tratamento da afta recorrente $e^{4,5-8}$. Quando empregado intermitentemente, por dois a três dias, ou em concentrações baixas, estimula ou restaura a imunidade celular ${ }^{9-11}$. Em concentrações altas, ou quando administrado por período prolongado, parece agir como imunossupressor ${ }^{12,13}$. N ormaliza a razão CD4/CD8, que se encon-

1. Professor titular do departamento de Otorrinolaringologia e Cirurgia de Cabeça e Pescoço, Universidade Federal de São Paulo (UNIFESP), São Paulo, SP

2. Professora assistente, chefe do setor de Estomatologia, departamentos de Dermatologia e Otorrinolaringologia, Universidade Federal de São Paulo (UNIFESP), São Paulo, SP

3. Pós-graduanda, setor de Estomatologia, departamentos de Dermatologia e Otorrinolaringologia, Universidade Federal de São Paulo (UNIFESP), São Paulo, SP: Chefe do Serviço de Dermatologia da Universidade do Oeste Paulista (UNOESTE), Presidente Prudente, SP

4. Professora assistente, setor de Estomatologia, departamentos de Dermatologia e Otorrinolaringologia, Universidade Federal de São Paulo (UNIFESP), São Paulo, SP

5. Professora de Epidemiologia, Universidade do Estado de Santa Catarina (UDESC), Chapecó, SC 
tra diminuída em pacientes com afta recorrente, e diminui os níveis séricos de IgA, IgM, interleucina-6 e interleucina-8 que podem estar aumentados nesses pacientes ${ }^{14-16}$.

O levamisol, para prevenção da afta recorrente, é usualmente prescrito por um período de 2 a 12 meses, na dose de 150 mg por dia por um, dois ou três dias consecutivos, a cada uma ou duas semanas, ou no início de um novo episódio de aftas 5,8,17-29. Em vários estudos, alguns destes utilizando ensaios com controle de placebo e/ou análise estatística, levamisol mostrou resultados favoráveis com respeito à prevenção de novos episódios $21,24,25,28,29$, diminuição do número das lesões ${ }^{17,21,24}$, duração das crises $^{17,21,24-26,28}$, tamanho das lesões $21,22,24,25$, intensidade da dor 21,22,24,29, ou resultando numa autoavaliação favorável do paciente ${ }^{23}$. Numa revisão da literatura em 1980, Miller $^{30}$ relatou resultados favoráveis $(62 \%$ a $100 \%$ ) com levamisol nos estudos abertos ou em estudos sem placebo; mas nos estudos duplos-cegos com placebo, apenas $50 \%$ dos pacientes apresentaram melhora clínica contra os outros $50 \%$ que não relataram melhora.

O levamisol é uma droga segura. Seus efeitos colaterais são leves e ocorrem em cerca de $10 \%$ dos $\operatorname{casos}^{30}$. Os mais frequentes são disgeusia, hiperosmia e náuseas; menos frequentemente os pacientes apresentam vômitos, diarreia, sonolência, rash cutâneo, cefaléia, insônia e excitação $0^{5,8,18,20,22,26-28}$.Entretanto, 0 uso do levamisol se tornou restrito devido à possibilidade de causar neutropenia ou agranulocitose ${ }^{11,31}$. Esse risco é muito baixo, mas aumenta em pacientes que recebem cursos prolongados de terapia diária e em pacientes com doença debilitante ou em uso concomitante de outras drogas tal como corticosteroides $^{32-34}$. O objetivo deste estudo foi utilizar um protocolo duplo-cego para avaliar a segurança e a efetividade do levamisol no tratamento profilático da afta recorrente.

\section{Métodos}

Foi realizado um ensaio clínico randomizado, duplo-cego e controlado por placebo com uma amostra de 28 pacientes portadores de afta recorrente, atendidos consecutivamente no ambulatório do setor de Estomatologia da Universidade Federal de São Paulo/Escola Paulista de M edicina. Pacientes portadores de afta minor, de etiologia desconhecida, e com pelo menos um episódio mensal de afta durante os últimos 12 meses foram incluídos. Para tanto, foram formados dois grupos: o grupo $\mathrm{A}$ recebeu levamisol e o grupo B recebeu placebo. Cada grupo contou inicialmente com 14 pacientes alocados aleatoriamente por sorteio.

Os critérios de inclusão foram ser portador de afta recorrente da forma minor, de etiologia desconhecida, com pelo menos um episódio mensal de afta durante os últimos 12 meses e os de exclusão foram pacientes com contraindicações para o uso do levamisol ${ }^{32-34}$ e gestantes.

Durante os seis meses do período do estudo, os pacientes do grupo A receberam $150 \mathrm{mg}$ de levamisol por via oral às segundas, quartas e sextas-feiras nas primeiras quatro semanas; em semanas alternadas da $5^{\text {a }}$ à $12^{\text {a }}$ semana; e a cada três semanas da $13^{\text {a }}$ à $24^{\text {a }}$ semana. Os pacientes do grupo B receberam uma medicação placebo no mesmo esquema posológico do grupo $A$. Todos os pacientes eram cegos à droga utilizada e não apresentavam aftas no início do tratamento, assim como o médico também não sabia para quais pacientes havia a prescrição de placebo. Um único médico, cego à composição dos grupos,

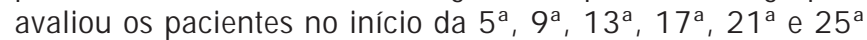
semana do tratamento.

Foram investigadas as seguintes variáveis: frequência dos episódios de aftas por mês; número de lesões por crise; duração em dias de cada episódio; dor; efeitos colaterais; e avaliação médica. Em cada avaliação os pacientes traziam anotações em um diário sobre a evolução de cada uma das variáveis estudadas. Para o critério da dor foi usado uma escala visual proposta por Souza e Silva em $2005^{35}$. O médico investigador registrava os efeitos colaterais relatados pelos pacientes e a contagem de células sanguíneas foi realizada a cada oito semanas. Após a avaliação final, o médico investigador classificou a condição clínica de cada paciente como melhorada ou inalterada.

Os participantes deste estudo estavam cientes dos métodos, da sua liberdade de escolha e concordaram em participar.

Para a análise estatística dos resultados foram usados testes não-paramétricos, levando-se em consideração a natureza das distribuições dos valores das variáveis com as seguintes escolhas: a) Análise de variância por postos de Friedman ${ }^{36}$, para comparar, separadamente, para o grupo A e para o grupo B, os valores do número dos episódios mensais de aftas, o número de lesões por crise, a duração das lesões e a contagem de células sanguíneas nos diferentes períodos de tempo. Quando houve diferença estatisticamente significante, a análise foi complementada pelo teste de comparações múltiplas ${ }^{37}$.

b) Teste de Mann-Whitney para duas amostras independentes $^{36}$, para comparar o grupo A com o grupo B, durante cada período do estudo, examinando os mesmos parâmetros citados no parágrafo anterior.

c) Teste Exato de Fisher para $2 \times 2^{36}$, para comparar os efeitos colaterais dos dois grupos. Essa comparação foi feita em separado para a 5a e 9 a semana do estudo. Foi usado 0 mesmo teste para comparar a intensidade da dor, nos grupos levamisol e placebo em cada período, e a avaliação final do médico investigador (percentagem de pacientes com melhora clínica).

d) Teste $G$ de Cochran ${ }^{36}$, para estudar as discordâncias da intensidade da dor, em cada período do estudo. Esse teste foi aplicado separadamente para cada um dos dois grupos. Quando houve discordância significante, complementamos com o teste de $\mathrm{McN}$ emar ${ }^{38}$, para comparar a presença da dor em cada período em relação ao período inicial.

A hipótese de nulidade em todos os testes teve como rejeição 0,05 (alfa < 0,05).

A preocupação inicial deste estudo foi demonstrar a hipótese da adequação da medicação à afta recorrente e, portanto, preocupamo-nos mais com a possibilidade do erro alpha.

\section{Resultados}

Dos 28 participantes iniciais, o grupo A concluiu o estudo com 14 indivíduos e o grupo B com 10 . 0 grupo A constituiuse de nove homens e de cinco mulheres, com idade entre 17 e 83 anos (média de 39 anos). 0 grupo B constituiu-se de oito homens e de duas mulheres, entre as idades de 12 e 46 anos (média de 26 anos). Os dois grupos de pacientes tinham 
WeCKX LLM ET AL.

padrão homogêneo das aftas antes de iniciar o tratamento, com diferença estatística apenas na duração das lesões, maior no grupo B (Tabelas 1 e 2; Quadro 1).

O número de episódios de aftas detectado nas avaliações mensais pós-tratamento dos grupos A e B são mostrados no Gráfico 1. Embora as médias sugiram tendência à diminuição do número de episódios mensais em ambos os grupos, a análise estatística não mostrou diferenças significantes $(p>0,05$, Friedman), assim como não houve diferenças entre os grupos em cada período do tratamento.

O número de lesões em cada episódio de afta, durante os períodos de avaliação, para os grupos $A$ e B, são mostrados no Gráfico 2. A análise estatística mostrou diminuição significante no número de lesões em ambos os grupos ( $p<0,05$, Friedman). No grupo $A$ isso foi evidente na $5 \underline{a}, 9 \underline{a}$, $13^{a}$ a e $17 \underline{a}$ semana do tratamento, enquanto no grupo B ocorreu na 17 a, 21 a e 25 a semana. A análise estatística não revelou diferenças significantes na comparação entre os grupos, durante o mesmo período de tratamento ( $p>0,05$, Mann-Whitney).

A duração das lesões, durante os episódios de afta para os grupos A e B, nos vários períodos do estudo, são mostradas no Gráfico 3. Houve diminuição na duração das lesões em sete dos pacientes do grupo A ( $50 \%$ ) e em oito dos pacientes do grupo B $(80 \%)$. A análise estatística mostrou diferença significante apenas no grupo B, no qual a duração das lesões foi significantemente menor na 17â , 21 a e 25a semana do tratamento ( $p<0,05$, Friedman). Na comparação dos dois grupos, durante o mesmo período de tempo, a diferença foi significante apenas antes de iniciar 0 tratamento quando o grupo $B$ mostrou maior duração das lesões ( $p<0,05$, Mann-Whitney). Embora tenha havido número médio menor de dias no grupo $B$ comparado ao grupo $A$, durante o tratamento, a diferença não foi estatisticamente significante.

O Gráfico 4 mostra os resultados da avaliação do parâmetro dor, durante as crises de afta, em cada período de avaliação para os dois grupos. Houve diminuição significante na intensidade da dor em ambos os grupos: no grupo A, a diminuição da dor ocorreu na 9a, 17 a e 25 a semana do tratamento, enquanto no grupo B ela ocorreu na 9ạ , 13aㅡ, 17aㅡ, 21a e 25a semana ( $p<0,05$, Cochran). Na comparação dos dois grupos, durante o mesmo período de tempo, os níveis da dor no grupo $B$ foram significantemente menor do que no grupo A, a partir da 13 a da semana do tratamento ( $p=0,0039$ na 13 a semana, $p=0,0020$ na 17 a, 21 a e 25 a semanas, McNemar).

A Tabela 4 mostra os resultados da avaliação final do médico investigador na 25a semana do tratamento. No grupo A, a melhora clínica ocorreu em $50 \%$ dos 14 pacientes, enquanto no grupo $\mathrm{B}$ a melhora ocorreu em $70 \%$ dos 10 pacientes; esta diferença não foi estatisticamente significante ( $p=0,289$, McNemar).

A incidência de efeitos colaterais na 5 a semana foi de $14 \%$ no grupo A (um paciente com azia e um com fraqueza) e de $20 \%$ no grupo B (um com azia e um com epigastralgia). Na 9 a semana do tratamento, um paciente relatou diarreia no grupo $A$. A partir da 9a semana, nenhum efeito colateral foi relatado nos dois grupos. Na comparação entre os grupos, pelo teste Exato de Fisher, a análise estatística não mostrou diferença significante na frequência de efeitos colaterais ( $p=0,5632$ na 5 a semana; $p=0,5833$ na 9 a semana). Não ocorreu neutropenia ou
Tabela 1 - Distribuição dos tratamentos segundo o sexo

\begin{tabular}{lccccc}
\hline Sexoltratamento & Levamisol & $\%$ & Placebo & $\%$ & Total \\
\hline Masculino & 5 & 35,7 & 2 & 20 & 7 \\
Feminino & 9 & 64,3 & 8 & 80 & 17 \\
Total & 14 & 100 & 10 & 100 & 24 \\
\hline
\end{tabular}

Tabela 2 - Medidas descritivas da idade segundo a droga

\begin{tabular}{lccc}
\hline Droga & N & Média & Desvio-padrão \\
\hline Levamisol & 14 & 39,1 & 15,6 \\
Placebo & 10 & 25,9 & 7,6 \\
Total & 24 & & \\
\hline
\end{tabular}

\begin{tabular}{lcccc}
\hline \multirow{2}{*}{ Tabela 3} & $\begin{array}{c}\text { - Avaliação final do médico investigador na } \\
\text { do tratamento dos grupos levamisol e placebo }\end{array}$ \\
\hline Grupo & $\begin{array}{c}\text { Melhora } \\
\text { clínica }\end{array}$ & Inalterado & Total & $\begin{array}{c}\% \\
\text { Melhora clínica }\end{array}$ \\
& 7 & 7 & 14 & 50,0 \\
Levamisol & 7 & 3 & 10 & 70,0 \\
Placebo & 14 & 10 & 24 & 58,3 \\
Total & Teste exato de Fisher (Levamisol x Placebo) $p=0,2896$ ou $28,96 \%$ &
\end{tabular}

Teste exato de Fisher (Levamisol x Placebo) $p=0,2896$ ou $28,96 \%$

agranulocitose, e a análise estatística não revelou diferenças significantes na contagem de células sanguíneas nos dois grupos durante todo o tratamento, nem na comparação entre ambos em cada período estudado.

\section{Discussão}

Inúmeras drogas têm sido utilizadas para o tratamento da afta recorrente. Entretanto, até o presente, faltam resultados consistentes que indiquem um medicamento efetivo e seguro, principalmente no que se refere à sua prevenção. O levamisol, por ser um eficiente restaurador da resposta imunológica celular, tem sido utilizado na prevenção da afta recorrente $e^{5,10,11,17-29}$.

Todos os estudos que utilizaram levamisol para tratamento da afta recorrente foram revisados, observando-se, entre eles, diversidade dos desenhos de estudo, da posologia e da dosagem. Devido a isso, para a realização deste estudo, foram elencados os trabalhos que apresentam metodologia similar, apresentados no Quadro 2. Foi feita uma sistematização dos achados em relação às variáveis que foram apresentadas nos resultados deste estudo, para facilitar a discussão.

Na metanálise dos estudos duplos-cego com uso de placebo de Lehner et al. ${ }^{17}(1976)$, M eyer et al..21(1977), Van de Heyning $(1978)^{24}$ e De Cree et al. ${ }^{28}$ (1978), incluindo este estudo, pode se observar que existe homogeneidade nos resultados obtidos em relação à duração das lesões, comprovando que não existe diferença significativa no tempo de duração das lesões com 0 uso do levamisol ( $p=0,1324$ para $G$ ). Para o número de lesões, a metanálise dos estudos de Weckx et al.(2008) e Meyer et al. $(1977)^{21}$, mostra também igualdade de resultados no uso do placebo e do levamisol $(p=0,2292)$. 


\begin{tabular}{lccc}
\hline \multicolumn{4}{c}{ Quadro 1 - Características clínicas dos pacientes antes do tratamento } \\
\hline Aspecto observado & Levamisol & Placebo & \\
\hline Média do número de episódios mensais de aftas & 2,2 & 2 & Teste de Mann-Whitney $p=0,9068$ \\
Média do número de lesões & 2,9 & 2,9 & Teste de Mann-Whitney $p=0,9068$ \\
Media do tempo de duração das lesões (dias) & 12,5 & 16,4 & Teste de Mann-Whitney $p=0,8606$ \\
Paciente com dor moderada ou intensa & 12 & 9 & Teste Exato de Fisher $p=0,7852$ \\
\hline
\end{tabular}

\section{Gráfico 1 - Episódios mensais de aftas durante 0 tratamento}

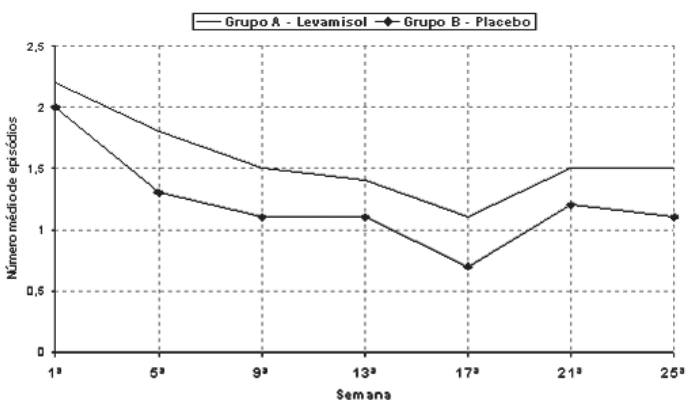

A nálise de variância por postos de Friedman

$\left(0 \times 5^{2} \times 9^{2} \times 13^{2} \times 17^{2} \times 21^{\circ} \times 25^{4}\right)$

$\chi^{2}$ 柆 $=12,59$

\begin{tabular}{|c|c|c|c|}
\hline \multicolumn{3}{|l|}{ Levamisol } & Placebo \\
\hline$\chi_{\text {calc }}^{2}=9,31$ & & & $\chi_{\text {calk }}^{2}=9,29$ \\
\hline \multicolumn{4}{|c|}{ Teste de Mann-Whitney (Levamisol x Placebo) } \\
\hline & Semana & Ucalc & Ucrítico \\
\hline & 0 & 68,0 & 41 \\
\hline & $5^{2}$ & 57,5 & \\
\hline & $\begin{array}{c}9^{2} \\
13^{2}\end{array}$ & $\begin{array}{l}56,0 \\
58,5\end{array}$ & \\
\hline & $17^{2}$ & 55,0 & \\
\hline & $21^{2}$ & 55,5 & \\
\hline & $25^{2}$ & 58.5 & \\
\hline
\end{tabular}

\section{Gráfico 3 - Tempo de duração das lesões de aftas durante o tratamento}

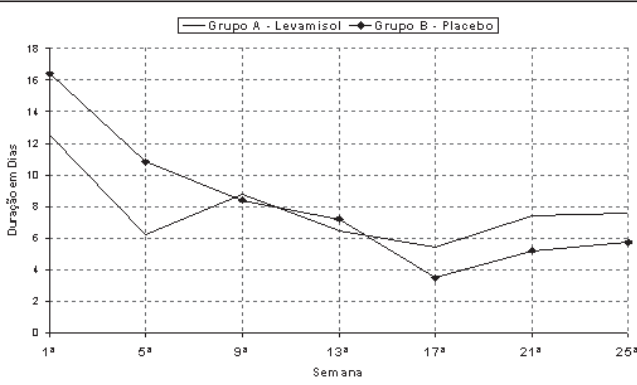

Análise de variância por postos de Friedman

$\left(0 \times 5^{\mathrm{a}} \times 9^{\mathrm{a}} \times 13^{\mathrm{a}} \times 17^{\mathrm{a}} \times 21^{\mathrm{a}} \times 25^{\mathrm{a}}\right)$

$$
\begin{aligned}
& \frac{\text { Levamiso1 }}{\chi_{\text {calc }}^{2}=11,68}
\end{aligned}
$$

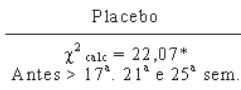

Teste de Mann-Whitney (Levamisol x Placebo)

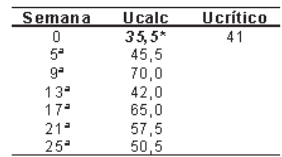

\section{Gráfico 2 - Número de lesões por crise durante o tratamento}

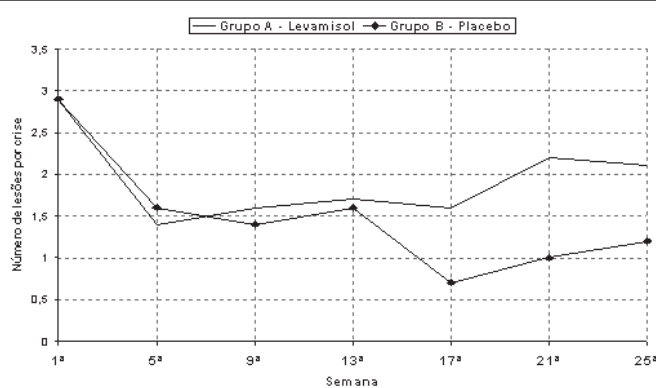

A nálise de variância por postos de Friedman

$\left(0 \times 5^{2} \times 9^{2} \times 13^{2} \times 17^{2} \times 21^{2} \times 25^{2}\right)$

$x_{\text {वrt }}^{2}=12,59$

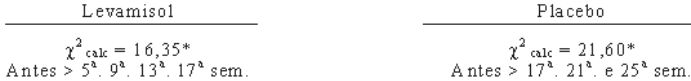

Teste de Mann-Whitney (Levamiso 1 x Placebo)
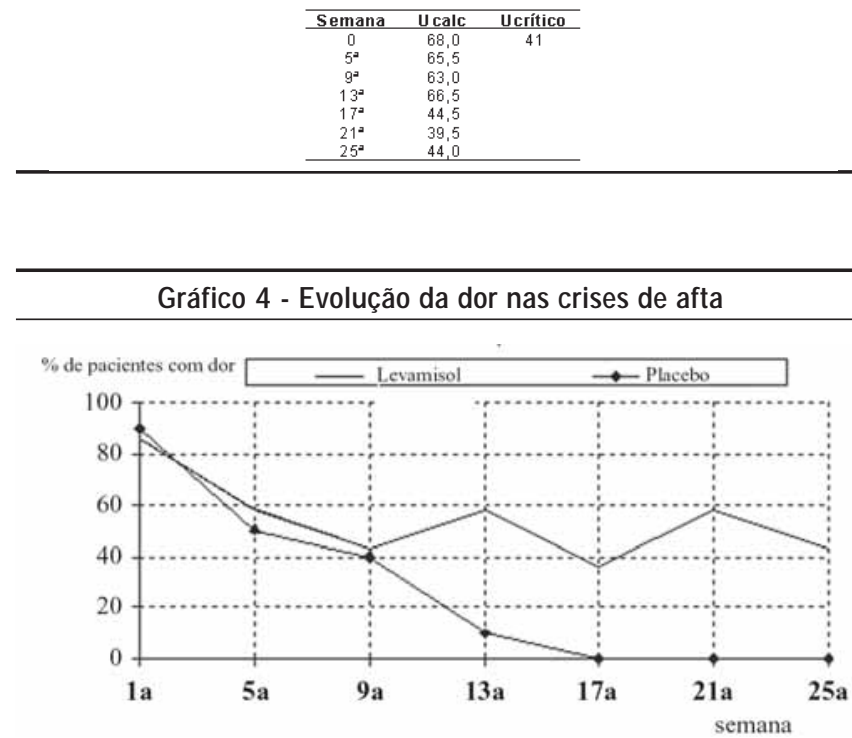

Teste G de Cochran

$\left(0 \times 5^{a} \times 9^{a} \times 13^{a} \times 17^{a} \times 21^{a} \times 25^{a}\right.$ semanas $)$ 


\begin{tabular}{|c|c|c|c|c|c|c|c|c|c|c|}
\hline \multirow[b]{2}{*}{ Autor e local } & \multirow[b]{2}{*}{$\begin{array}{c}\text { Ano de } \\
\text { publicação }\end{array}$} & \multirow[b]{2}{*}{$\begin{array}{c}\mathrm{N}^{0} \text { de } \\
\text { pacientes }\end{array}$} & \multirow[b]{2}{*}{ Posologia } & \multirow[b]{2}{*}{$\begin{array}{l}\text { Seguimento } \\
\text { (em meses) }\end{array}$} & \multicolumn{6}{|c|}{ Diferença significante a favor do levamisol: Sim ou Não } \\
\hline & & & & & $\begin{array}{l}\text { Frequência } \\
\text { das crises }\end{array}$ & $\begin{array}{l}\text { Duração } \\
\text { da crise }\end{array}$ & $\begin{array}{l}\text { Intensidade } \\
\text { da dor }\end{array}$ & $\mathrm{N}^{0}$ de lesões & $\begin{array}{l}\text { Tamanho } \\
\text { das lesões }\end{array}$ & $\begin{array}{l}\text { Opinião do } \\
\text { investigador }\end{array}$ \\
\hline $\begin{array}{l}\text { Lehner et al. } \\
\text { (Londres) }\end{array}$ & 1976 & 47 & $\begin{array}{c}F \\
2 m\end{array}$ & 6 & - & Sim & Não & Sim & - & - \\
\hline $\begin{array}{l}\text { Meyer et al. } \\
\text { (Bélgica) }\end{array}$ & $\begin{array}{l}1977 \\
1977\end{array}$ & $\begin{array}{l}62 \\
13\end{array}$ & $\begin{array}{c}\mathrm{A} \\
4 \mathrm{~m} \\
\mathrm{D}\end{array}$ & $\begin{array}{l}4 \\
4\end{array}$ & $\begin{array}{l}\text { Sim } \\
\text { Não }\end{array}$ & $\begin{array}{l}\text { Sim } \\
\text { Sim }\end{array}$ & $\begin{array}{l}\text { Sim } \\
\text { Sim }\end{array}$ & $\begin{array}{l}\text { Sim } \\
\text { Sim }\end{array}$ & $\begin{array}{l}\text { Sim } \\
\text { Sim }\end{array}$ & - \\
\hline $\begin{array}{l}\text { De Cree et al. } \\
\text { (Bélgica) }\end{array}$ & 1978 & 18 & B & 3 & Sim & Sim & Não & - & - & - \\
\hline $\begin{array}{l}\text { Drinnan, Fischman } \\
\text { (N. York) }\end{array}$ & 1978 & 24 & B & 6 & Não & Não & - & - & - & Não \\
\hline $\begin{array}{l}\text { Miller et al. } \\
\text { (Pensilvânia) }\end{array}$ & 1978 & 20 & $\begin{array}{c}A \\
2 \text { a } 5 \mathrm{~m}\end{array}$ & 2 a 5 & Não & Não & - & - & - & - \\
\hline $\begin{array}{l}\text { Olson, Silverman } \\
\text { (Califórnia) }\end{array}$ & 1978 & 48 & B & 6 crises & Não & Não & Não & - & - & Sim \\
\hline $\begin{array}{l}\text { Van De Heyning } \\
\text { (Bélgica) }\end{array}$ & 1978 & 13 & $\begin{array}{c}A \\
2 \mathrm{~m}\end{array}$ & 4 & Sim & Não & Não & Sim & - & - \\
\hline $\begin{array}{l}\text { Luderschmidt } \\
\text { (Alemanha) }\end{array}$ & 1979 & 17 & $E$ & $?$ & Não & Não & Não & - & - & - \\
\hline $\begin{array}{l}\text { Zissis et al. } \\
\text { (Grécia) }\end{array}$ & $\begin{array}{l}1983 \\
1983\end{array}$ & 33 & $\begin{array}{c}F \\
4 \mathrm{~m} \\
\mathrm{G}\end{array}$ & $\begin{array}{l}4 \\
4\end{array}$ & $\begin{array}{l}\text { Sim } \\
\text { Sim }\end{array}$ & $\begin{array}{l}\text { Sim } \\
\text { Sim }\end{array}$ & $\begin{array}{l}\text { Sim } \\
\text { Sim }\end{array}$ & - & - & - \\
\hline Weckx et al. (Brasil) & ) 2008 & 14 & $H$ & 6 & Não & Não & Não & Não & - & Não \\
\hline
\end{tabular}

Posologia do Levamisol (m: meses)

A- $150 \mathrm{mg}$ ao dia, por três dias, a cada duas semanas.

B- $150 \mathrm{mg}$ ao dia, por três dias, a cada nova crise.

C- $150 \mathrm{mg}$ ao dia, por dois dias, duas semanas sim, duas semanas não.

D- $150 \mathrm{mg}$ ao dia, por três dias, a cada duas semanas, por dois meses e depois só na recidiva.

E- $150 \mathrm{mg}$ ao dia, por três dias, durante seis semanas.

F- $150 \mathrm{mg}$ ao dia, por dois dias, a cada semana.

G- $150 \mathrm{mg}$ ao dia, por um dia, a cada semana, 4 meses.

H- 150 mg ao dia, por três dias nas primeiras 4 semanas; em semanas alternadas da $5^{\text {a }}$ à $12^{\text {a }}$ semana; e a cada três semanas da $13^{\mathrm{a}}$ à $24^{\mathrm{a}}$ semana.

A maioria dos autores (Quadro 2) observou frequência de efeitos colaterais em torno de $15 \%$ a $33 \%^{10,13,25-28}$, porém os efeitos colaterais observados neste estudo foram raros e de intensidade leve, sem ocorrência de neutropenia, conforme observado por Graber et al. ${ }^{33}(1976)$, Vanholder, Van Hove $(1977)^{34}$, de Souza et al. $(1980)^{32}$ e Machado et al. (1982) $)^{11}$, não obrigando a interrupção do tratamento ou o parcelamento da dose, em concordância com os autores Verhaegen et al. $(1973)^{5}$, Symoens, Brugmans $(1974)^{20}$, Carvalho et al. $(1976)^{8}$, Meyer et al. $(1977)^{22}$, Kaplan et al. $(1978)^{18}$, Gier et al. $(1978)^{26}$, Drinnan, Fischman $(1978)^{27}$, De Cree et al. $(1978)^{28}$ e Zissis et al. $(1983)^{25}$, que empregaram levamisol para tratar pacientes com afta recorrente.

A literatura mostra resultados que divergem entre si. Assim, os estudos iniciais de Verhaegen et al. $(1973)^{5}$, Carvalho et al.
$(1976)^{8}$, Machado $(1981)^{10}$, Machado et al.. $(1982)^{11}$, Symoens, Brugmans $(1974)^{20}$ e Van de Heyning $(1978)^{24}$, que empregaram levamisol sem o controle de placebo, mostraram $62 \%$ a $100 \%$ dos pacientes tratados com melhora ou cura. Por outro lado, quando os estudos usam métodos mais rigorosos, como os duplos-cegos, e com análise estatística de resultados, comparando grupos com placebo, se revelam tanto resultados favoráveis $17,21,24,25,28$ quanto sem melhora significante $22,23,27$. Mesmo nos estudos duplos-cegos de Kaplan et al. $(1978)^{18}$ e de Gier et al. $(1978)^{26}$, mas sem análise estatística para os resultados, pôde-se verificar conclusões divergentes frente ao levamisol. Quando Meyer et al. (1977) ${ }^{21}$ e Haneke (1979) ${ }^{29}$ conduziram estudos clínicos, sem controle de grupo placebo, relata-se melhora ao final do tratamento com levamisol.

Assim, se forem comparados este estudo com os dados da literatura, há concordância com os estudos iniciais sem 
placebo5,8,10,11,20,24, com cura ou melhora praticamente para todos os pacientes. Esse efeito se dá, provavelmente, como observado no atual estudo, devido à notável interferência do efeito placebo nos desfechos ${ }^{39}$. Esses estudos avaliaram casuísticas semelhantes à esta ${ }^{5,8,10,11,24}$, exceto Symoens e Brugmans, em $1974^{20}$, que em um estudo multicêntrico avaliou número maior, incluindo 82 pacientes.

Por outro lado, se forem comparados os resultados deste estudo a outros feitos com métodos semelhantes - esquemas posológicos, duplos-cegos e com análise estatística - encontram-se resultados concordantes com alguns ${ }^{22,23,27}$, mas contrários a outros ${ }^{17,21,24,25,28}$. Assim, Miller et al., em 1978 ${ }^{22}$, observaram apenas melhora dos sintomas subjetivos com levamisol. De forma similar, Olson, Silverman, em 197823, observaram diferença significante apenas no critério opinião do paciente, a favor da droga ativa. Drinnan, Fischman (1978) ${ }^{27}$, ainda mais próximos aos achados deste estudo, obtiveram vantagem para o placebo, porém, seu método de administrar o medicamento foi somente a cada novo episódio de afta. Contrariando esses achados, os estudos duplos-cego com análise estatística de Lehner et al. $(1976)^{17}$, Meyer et al. (1977) $)^{21}$, Van de Heyning $(1978)^{24}$, Zissis et al. $(1983)^{25}$ e De Cree et al. $(1978)^{28}$ mostraram que o levamisol é uma droga eficaz nos diversos esquemas posológicos, mesmo em doses mínimas.

Pode-se atribuir algumas explicações para essas diferenças de resultados, além da presença ou da ausência de um grupo controle, sendo salientados os objetivos diferentes de cada estudo, o caráter subjetivo da gravidade da úlcera ou as posologias diversas empregadas. Embora a dosagem diária aqui utilizada tenha sido equivalente a outros estudos, o tempo de emprego durante a semana diferiu de alguns autores $^{5,10,17,25}$, e a dose total semanal foi diferente da de todos os estudos revisados. Esta casuística incluiu somente casos de afta minor enquanto outros autores incluíram também afta major ${ }^{5,10}$.

É necessário enfatizar a melhora importante daqueles pacientes que receberam placebo, fato relatado na literatura, que pode ser explicado pela autossugestão e pela expectativa do paciente. ${ }^{39}$ Isso ocorre porque os fatores emocionais podem interferir na evolução das aftas recorrentes, o que foi mencionado por muitos pacientes deste estudo e também é descrito na literatura 22,23,25-28.

Este estudo reforça o conceito de que quando há componentes psicossomáticos, a investigação terapêutica deverá utilizar um desenho de estudo controlado por placebo, já que o placebo pode muitas vezes causar efeitos benéficos.

\section{Conclusões}

Com base nos resultados desse estudo duplo-cego, controlado por placebo, de levamisol para tratamento da afta recorrente, concluiu-se que:

1. Levamisol, como usado nesse protocolo, é uma droga segura.

2. Comparado ao placebo, levamisol não é efetivo no tratamento profilático da afta recorrente.

3. O efeito placebo é importante em desordens nas quais fatores emocionais afetam a recorrência ou a expressão de sintomas.

\section{Agradecimentos}

Os autores agradecem à enfermeira Laurentina Rodrigues Freitas pela sua ajuda na distribuição dos medicamentos aos pacientes.

\section{Conflito de interesse: não há}

\section{SUMMARY}

LEVAMISOLE DOES NOT PREVENT LESIONS OF RECURRENT APHTHOUS STOMATITIS: A DOUBLE-BLIND PLACEBO-CONTROLLED CLINICAL TRIAL

Objective. to utilize a double-blind protocol to provide clarification about the safety and effectiveness of levamisole in the treatment of recurrent aphthous stomatitis.

Methods. Fourteen patients took a decreasing dose of oral levamisole for six months (initial dose $150 \mathrm{mg}$ three times a week) and ten others were placebo control patients. All were evaluated monthly.

RESULTS. The number of crises had a tendency to decrease in both groups, but without a difference between groups. The number of lesions diminished significantly in the two groups, but upon comparison the difference was not significant. Duration of the lesions diminished significantly in the placebo, however when compared to the levamisole group, difference was not significant during treatment. The intensity of pain was significantly lower in the two groups, but upon comparison, pain was significantly lower in the placebo group. The final global evaluation showed improvement in $50 \%$ of patients of the levamisole group and in $70 \%$ of the placebo, without a significant difference between treatments. No difference in the frequency of collateral effects was observed between groups.

Conclusions. Levamisole, as used in this protocol, is a safe drug. When compared with the placebo, levamisole is not effective in the prophylactic treatment of recurrent aphthous stomatitis. The placebo effect is important in diseases where emotional factors affect recurrence or expression of symptoms. [Rev Assoc Med Bras 2009; 55(2): 132-8]

KeY Words: Levamisole. Placebo effect. Randomized controlled trial. Aphthous stomatitis. Therapy.

\section{ReferênCIAS}

1. Ship J A. Recurrent aphthous stomatitis. An update. Oral Surg Oral Med Oral Pathol Oral Radiol Endod. 1996;81:141-7.

2. Zunt SL. Recurrent aphthous stomatitis. Dermatol Clin. 2003;21:33-9.

3. Woo SB, Sonis ST. Recurrent aphthous ulcers: a review of diagnosis and treatment. J Am Dent Assoc. 1996;127:1202-13.

4. Symoens J, Rosenthal M. Levamisole in the modulation of the immune response: the current experimental and clinical state. J Reticuloendothel Soc. 1977;21:175-221

5. Verhaegen H, De Cree J, Brugmans J. Treatment of aphthous stomatitis [Letter] Lancet. 1973;2:842.

6. Renoux G, Renoux M, Teller MN, McMahon S, Guillaumin J M. Potentiation of T-cell mediated immunity by levamisole. Clin Exp Immunol. $1976 ; 25: 288-96$.

7. Brugmans J , Schuermans V, De Cock W, Thienpont D, J anssen P, Verhaegen $H$, et al. Restoration of host defense mechanisms in man by levamisole. Life Sci, 1973;13:1499-504.

8. Carvalho CQ, Otuki TK, Poli ME, Nogueira J L, Guerrero J. Levamisole no tratamento da afta oral recidivante e do herpes simples recorrente. An Bras Dermatol. 1976;51:115-25.

9. Rytel MW. Can therapeutic considerations provide clues to the etiology of aphthous stomatitis and Bheçets syndrome. J Oral Pathol. $1978 ; 7: 372-5$ 
WECKX LLM ET AL.

10. Machado J A. Levamisole - um novo tratamento para dois velhos problemas: ulceração aftosa recorrente e herpes simples recorrente labial. Rev Assoc Paul Cir Dent. 1981;35:20-6.

11. Machado J A, Moreira HW, Fonseca LM, Arantes JB. Avaliação clínica e hematológica do uso do levamisole no herpes simples recorrente e na afta oral recidivante. Odontol Mod. 1982;9:36-43

12. Sampson D, Lui A. The effect of levamisole on cell-mediated immunity and suppressor cell function. Cancer Res. 1976;36:952-5.

13. Arnold HL J r. Immunotherapy with levamisole [Letter] $\mathrm{N}$ Engl Med. $1976 ; 294: 447$

14. Sun A, Chiang CP, Chiou PS, Wang J T, Liu BY, Wu YC, Immunomodulation by levamisole in patients with recurrent aphthous ulcers or oral lichen planus. J Oral Pathol Med. 1994:23:172-7.

15. Sun A, Chia J S, Chang YF, Chiang CP. Levamisole and chinese medicinal herbs can modulate the serum interleukin- 6 level in patients with recurrent aphthous ulcerations. J Oral Pathol Med. 2003;32:206-14.

16. Sun A, Chang YF, Chia J S, Chiang CP. Serum interleukin-8 level is a more sensitive marker than serum interleukin-6 level in monitoring the disease activity of recurrent aphthous ulcerations. J Oral Pathol Med. 2004;33:133-9.

17. Lehner T, Wilton J M, Ivanyi L. Double blind crossover trial of levamisole in recurrent aphthous ulceration. Lancet. 1976;2:926-9.

18. Kaplan B, Cardarelli C, Pinnell SR. Double-blind study of levamisole in aphthous stomatitis. J Oral Pathol. 1978;7:400-4

19. Tommasi AF. Lesões ulcerativas e vésico-bolhosas. In: Tommasi AF, editor. Diagnóstico em patologia bucal. São Paulo: Artes Médicas; 1982. p.111-33.

20. Symoens ], Brugmans ]. [Letter] Treatment of recurrent aphthous stomatitis and herpes with levamisole. Br Med J. 1974;4:592.

21. Meyer J D, Degraeve M, Clarysse J, De Loose F, Peremans W. Levamisole in aphthous stomatitis: evaluation of three regimens. BrMed J . 1977;1:671-4.

22. Miller MF, Silvert ME, Laster LL, Green P, Ship II. Effect of levamisole on the incidence and prevalence of recurrent aphthous stomatitis. A double-blind clinical trial. J Oral Pathol. 1978;7:387-92.

23. Olson JA, Silverman S J r. Double-blind study of levamisole therapy in recurrent aphthous stomatitis. J Oral Pathol. 1978;7:393-9.

24. Van de Heyning J. Levamisole in the treatment of recurrent aphthous stomatitis. Laryngoscope. 1978;88:522-7

25. Zissis NP, Hatzioti AJ, Antoniadis S, Ninika A, Hatziotis J C. Therapeutic evaluation of levamisole in recurrent aphthous stomatitis. Double-blind comparison of two dosage schedules of levamisole and placebo. J Oral Med. $1983 ; 38: 161-3$
26. Gier RE, George B, Wilson T, Rueger A, HartJ K, Quaison F et al. Evaluation of the therapeutic effect of levamisole in treatment of recurrent aphthous stomatitis. J Oral Pathol. 1978;7:405-13.

27. Drinnan AJ , Fischman SL. Randomized double-blind study of levamisole in recurrent aphthous stomatitis. J Oral Pathol. 1978;7:414-7.

28. De Cree J, Verhaegen $H$, De Cock W, Verbruggen F. A randomized doubleblind trial of levamisole in the therapy of recurrent aphthous stomatitis. Oral Surg Oral Med Oral Pathol. 1978;45:378-84

29. Haneke E. Levamisol therapie bie verschiedenen dermatoogischen indikationen. Z Hautkr. 1979;54:408-14.

30. Miller MF. Use of levamisole in recurrent aphthous stomatitis. Drugs. $1980 ; 20: 131-6$

31. Bork K, Hoed N, Korting GW. Aftas de origem não infecciosa. In: Bork K Hoed N, Korting GW, editors. Doenças e sintomas da cavidade bucal e da região perioral. Atlas colorido. São Paulo: Manole; 1988. p.48-53.

32. de Souza CA, Lorand IG, Teles CM, de Lucca RS, Struckel LC. Agranulocitose por levamisol. Rev Assoc Med Brás. 1980;26:307-8

33. Graber H, Takacs L, Vedrody K. Agranulocytosis due to levamisole. Lancet. $1976 ; 2: 1248$

34. Vanholder R, Van Hove W. Recurrent agranulocytosis after levamisole. Lancet. 1977;1:100.

35. Sousa, F. F.; Silva, J. A. A métrica da dor (dormetria): problemas teóricos e metodológicos; 2005. Rev Dor, 6:469-513.

36. Siegel S, editor. Estadística no paramétrica. México: Trillas; 1975.

37. Hollander M, Wolfe DA, editors. Nonparametric statistical methods. New York: Wiley \& Sons; 1973

38. Remington RD, Schork MA, editors. Statistics with applications to the biological and health sciences. New J ersey: Prentice Hall, INC, Englewood Cliffs; 1970.

39. Macedo A, Farre M, Banos JE. Placebo effect and placebos: what are we talking about? Some conceptual and historical considerations. Eur J Clin Pharmacol. 2003:59:337-42.

Artigo recebido: 12/11/07 Aceito para publicação: 04/07/08 